\title{
Beyond Wavelet Transform based Image Denosing
}

\author{
Yujie Li ${ }^{*}$, Shiyuan Yang, Seiichi Serikawa \\ Kyushu Institute of Technology, 804-8550 Kitakyushu, Japan \\ *Corresponding Author: yzyjli@126.com
}

\begin{abstract}
In this paper, we proposed beyond wavelets transform based image denoising method. By using beyond wavelet transform, the noised image is firstly decomposed into some low frequency sub-bands and high frequency sub-bands. All the beyond wavelets coefficients of the original image are estimated by Bayesian MAP estimator rule. Experimental results show that the denoising effect is better than the other methods.
\end{abstract}

Keywords: Image denoising, Curvelet transform, Contourlet transform.

\section{Introduction}

Generally, because of the imaging devices and the external noise interference, digital images are often degraded during transmission and acquisition process. Image denoising is that how to separate the noise and useful information in a particular space area, so imaging denoising is an essential part in image restoration. Ordinary, the image denoising methods are roughly divided in two parts: spatial domain methods and transform domain methods. Spatial domain denoising is with the classical assumption, that is, the input noisy images are piecewise constant and the neighbor pixels are highly correlated. So, the spatial domain denoising is to filter the nearby pixels. On the other hand, the transform domain methods transform the image from the spatial domain into a different domain and suppress noise in the transform domain ${ }^{(1)}$.

Over the last decades, many of methods have been proposed for image denoising in transform domain, such as Wavelet Transform Denoising. Unlike the sine used in Fourier transform for decomposition of a signal, wavelets are generally much more concentrated in time. They usually provide an analysis of the signal which is localized in both time and frequency, whereas Fourier transform is localized only in frequency. Wavelet Transform can provide optimal representation for piecewise smooth signal of one-dimensional and it also has the tree decomposition algorithms ${ }^{(2)}$. Useful signal usually contain low-frequency signals and some stable signals, but noise is typically some high-frequency signals. First, the original signal is decomposed by wavelet; noise is contained in the high-frequency coefficients. Then the high-frequency coefficients of wavelet decomposition are quantified by threshold methods. In recent years, some researches have addressed the development of statistical models of Wavelet Domain Denoising, however, the main disadvantage of wavelets in two-dimensions or higher is their limited ability in capturing directional information. To overcome these shortcomings, some researchers have proposed multiscale and directional representations that can capture the geometrical structures very well. Such as Wedgelet Transform, Curvelet Transform, Contourlet Transform and so on. The most simple way of image denoising in the beyond wavelet transform domain is to threshold the beyond wavelet coefficients ${ }^{(3)}$. Recently, lots of approaches have received many attentions of researchers and have shown good performance in beyond wavelet image denoising methods.

In this paper, we proposed beyond wavelets transform ${ }^{(8)}$ based image denoising method. By using beyond wavelet transform, the noised image is firstly decomposed into some low frequency sub-bands and high frequency sub-bands. All the beyond wavelets coefficients of the original image are estimated by Bayesian MAP estimator rule. The organization of this paper is as follows. We summarize the recent beyond wavelets transform methods in Section 2. In Section 3, combined with Bayesian MAP estimator rule, beyond wavelets based image denoising method will be discussed. We apply the method in some images in Section 4. Finally, a conclusion is presented in Section 5 .

\section{Beyond Wavelets Methods}

Curvelet transform, Contourlet transform et al can overcome the shortcomings of Wavelet Transform and capture the directional information, so we can call them 
X-let Transform or Beyond Wavelet Transform ${ }^{(4)}$. In the below, we briefly introduce the principle of them.

\subsection{Curvelet Transform}

Curvelet transform is a non-adaptive technique for multi-scale object representation. It is being extended by the wavelet concept. They are becoming popular in image processing in recent years.

As a new multi-scale analysis method, curvelet transform is more suitable than wavelet in analysis of curve or straight edge features. It also has higher precision and better sparse approximation expression. It divided the image into sub-bands of different scales in different sub-bands by a filter, then the edge information and the noise information can be clearly separated, at last do a transformation for the each sub-band block ${ }^{(5)}$.

\subsection{Contourlet Transform}

In 2002, Do and Vetterli proposed an efficient directional multi-resolution image representation called contourlet transform. The main idea of it is that the basic function is approximated with line segments when it is approximation of the original image, in order to express the original image with little coefficients. Contourlet transform contains multiscale analysis and direction analysis. It is decomposed into low frequency sub-band and high frequency sub-band by LP, then be processed by directional filter banks. Contourlet Transform can capture the the direction signal of the image, it is a real two dimensional transform that can capture the intrinsic geometrical structure, and it also has been applied in many image processing. Because of its anisotropy and directionality, contourlet transform better represents the salient features of the image such as, edges, lines, curves, and contours, than wavelet transform. ${ }^{(6)}$

\section{Proposed Method}

Let us think that an image is corrupted by noise with variance $\sigma_{n}$. The image with noise can be decomposed by beyond wavelet transform to gain beyond wavelet coefficients. But, in many cases, it does not consider the relationship of the parent and the coefficient. In order to solve this problem, we proposed a new model for image denoising, which based on Bayesian MAP estimator rule ${ }^{(7)}$. The flowchart of our processing is presented in figure 1. First, the input noisy image is decomposed to beyond wavelet sub-bands, local neighborhood pixels are estimated by Bayesian MAP estimator rule, and then the new coefficients of reference pixels are gotten. At last, we do a reconstruction for beyond wavelet transform, the new beyond wavelet sub-bands can be got and the denoised image is acquired.

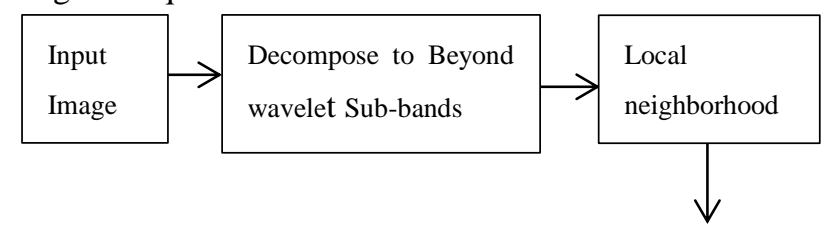

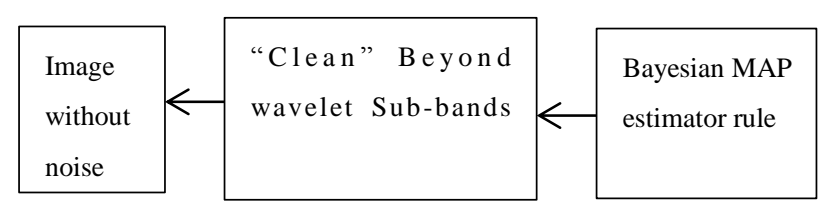

Figure 1. Flowchart of our proposed method for image denoising

First, let $g$ equally spaced samples of a real-valued image, $n$ is normal random variable also is independently and identically distribute. The image with noise $x$ can be expressed as

$$
x=g+n
$$

This problem can be formulated as the following equation in Curvelet transform domain

$$
y=s+n
$$

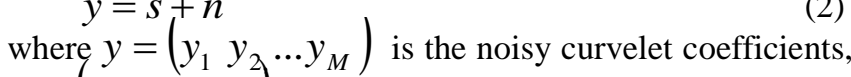
$s=\left(s_{1}, s_{2}, \ldots . s_{M}\right)$ is the noise-free coefficients, and $n=\left(n_{1}, n_{2}, \ldots n_{M}\right)$ is the independent noise. Take the standard MAP estimator for (2), we can obtain

$$
\hat{s}(y)=\arg \max _{s} p_{s \mid y}(s \mid y)
$$

By using the Bayes rule, the (3) is equivalent to

$$
\hat{s(y)}=\arg \max _{s}\left[p_{y \mid s}(y \mid s) p_{s}(s)\right]=\arg \max _{s} p_{n}(y-s) p_{s}(s)
$$

Take a logarithm to (4), then

$$
\hat{s}(y)=\arg \max \left[\log \left(p_{n}(y-s)\right)+\log \left(p_{s}(s)\right)\right]
$$

The spherically-contoured zero-mean $d$-dimensional Bessel $\mathrm{K}$ Function (BKF) density is

$$
p_{s}(s)=\frac{2}{(2 \pi c) \Gamma(p)}\left(\frac{\sqrt{2 c}}{\|s\|}\right)^{d / 2-p} K_{1-p}\left(\sqrt{\frac{2}{c}}\|s\|\right)^{(6)}
$$

where, $K_{1-p}(u)$ is the modified Bessel function. $c$ and $p$ are the scale parameter and shape parameter. In this paper, we propose a simple non-Gaussian multivariate PDF to model the noise-free coefficients, considering the relationship between a coefficient, neighbors, cousins and parent.

$$
p_{Y}(y)=\frac{\exp \left(\frac{\sigma_{n}^{2}}{c}\right)}{(2 \pi c) \Gamma(p)} \times \sum_{j=0}^{\infty}\left(-\frac{\sigma_{n}^{2}}{c}\right) \frac{(p-1)_{j}}{j !} \Gamma\left(p-\frac{d}{2}-j, \frac{\sigma_{n}^{2}}{c}, \frac{\|c\|^{2}}{2 c}\right) \text { (7) }
$$

with $j$ factor in both the numerator and denominator of the fraction. $\sigma_{n}$ is the standard deviation of the noise coefficients. $y=\operatorname{sqrt}\left(\left\|y_{i}\right\|^{2}+\left\|y_{i}^{[p]}\right\|^{2}+\left\|y_{i}^{[c]}\right\|^{2}\right) y_{i}$ and $y_{i}^{[p]}$ are dependent on each other, but the neighbors and cousins are independent on parent. Let us use the MAP estimator for this model. Maximizing the (5) for each component, we can get

$$
y_{i}=\hat{s_{i}}-\sigma_{n}^{2} \frac{d \log p_{s}(s)}{d \hat{s_{i}}}
$$

where, $i \in[1, d]$.the property of the modified Bessel function of the second kind $K_{\lambda}(u)$ is 


$$
\frac{d}{d u} \log K_{\lambda}(u)=\frac{\lambda}{u}-\frac{K_{\lambda+1}(u)}{K_{\lambda}(u)}
$$

Then, the second term of (8) can be computed as

$$
\frac{d}{d \hat{s_{i}}} \log p_{s}(s)=-\frac{\hat{s_{i}}}{\|\hat{A}\|} \sqrt{2 / c} \frac{K_{d / 2-p+1}(\sqrt{2 / c}\|\hat{s}\|)}{K_{d / 2}(\sqrt{2 / c}\|\hat{s}\|)}(10)
$$

Through the above equations, the MAP estimator can be formulated using (7) and (9), it gives

$$
y_{i}=\hat{s_{i}}\left[1+\frac{\sigma_{n}^{2}}{\|\hat{s}\|} \sqrt{2 / c} \frac{K_{d / 2-p+1}(\sqrt{2 / c}\|\hat{s}\|)}{K_{d / 2-p}(\sqrt{2 / c}\|\hat{s}\|)}\right]
$$

We approximate the $\|\hat{s}\|$ as $\|y\|$. The multivariate shrinkage function can be written as

$$
\hat{s}_{i} \approx \frac{y_{i}}{\left[1+\frac{\sigma_{n}^{2}}{\|y\|} \sqrt{2 / c} \frac{K_{d / 2-p+1}(\sqrt{2 / c}\|y\|)}{K_{d / 2-p}(\sqrt{2 / c}\|y\|)}\right]}
$$

where $\sigma_{n}=\operatorname{Median}\left(y_{i}\right) / 0.6748, p=3(\operatorname{Kurt}(X)-3)$, $c=\operatorname{Var}(X) / p \cdot X$ is the HH sub-bands. $\operatorname{Var}(X)$ and $\operatorname{Kurt}(X)$ are the variance and kurtosis.

\section{Experimental Results}

In this session, we show some experiments of image denoising with the proposed models described in the previous section. In order to do a simulation, we use the window XP, Intel Core Duo 2, 2.0GHz, 1 GB RAM memory computer to calculate. In the following experiments, we compare the proposed method with some other methods in details.

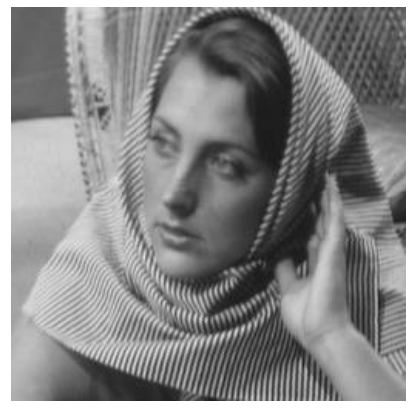

(a)

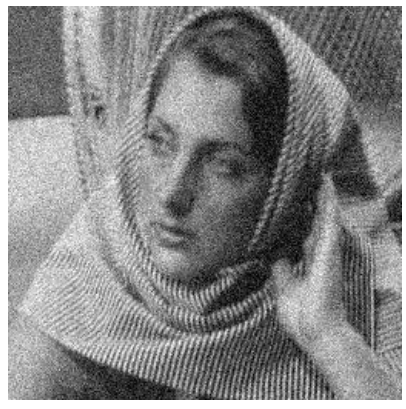

(b)

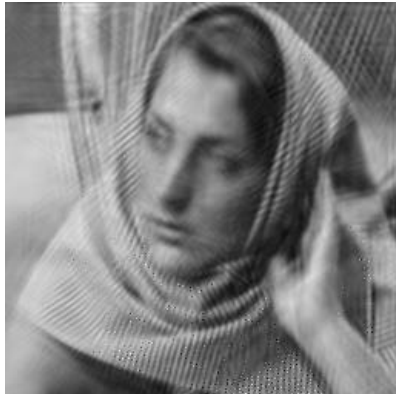

(c)

(e)

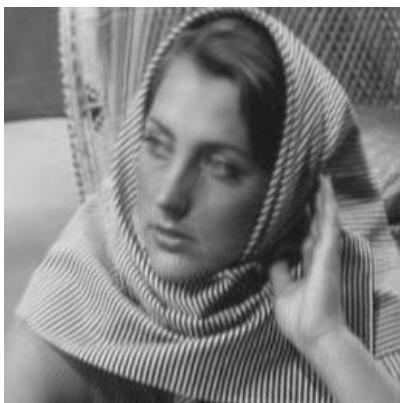

(g)

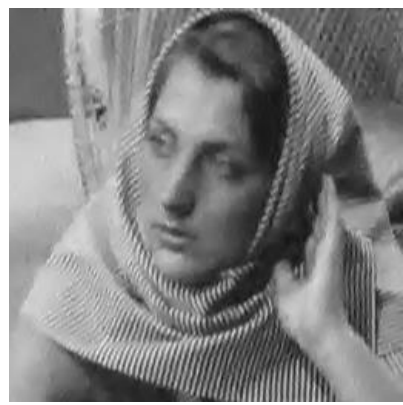

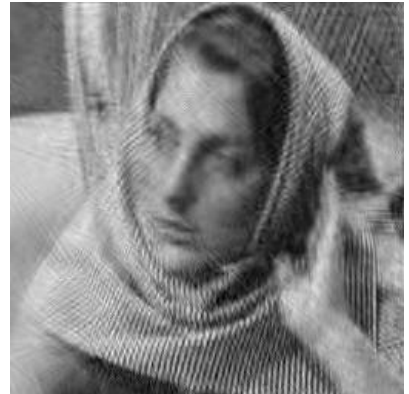

(d)

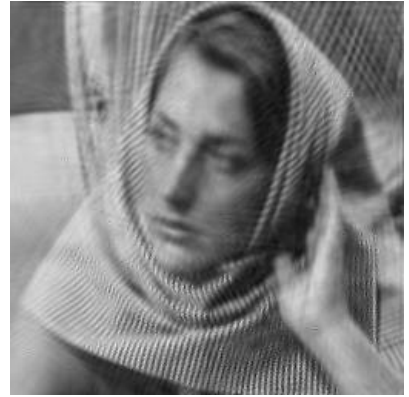

(f)

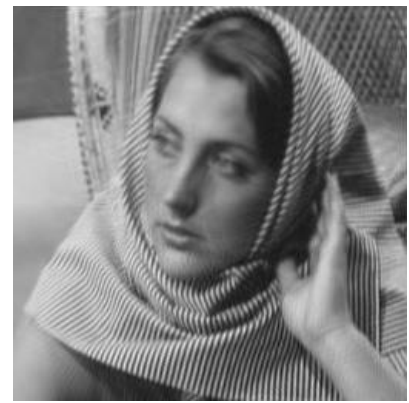

(h)
Figure2. Experimental results. (a)Barbara; (b) noisy $\left(\sigma_{n}=20\right)$; (c) denoised with $\mathrm{K}_{-}$Sigma $_{1}$; (d) denoised with $\mathrm{K}$-Sigma 2 ;(e) denoised with BLS-GSM;(f) denoised with GSCE; (g) denoised with curvelet and Bayesian MAP estimator rule;(h) denoised with contourlet and Bayesian MAP estimator rule

In figure2, we compare the Barbara image denoising results with other methods. (g)and(h) are the results with our proposed methods. From the below table, we can find obviously that, the proposed method is outperforming. The value of PSNR is highest than the others, which is the higher the better. The average improvement in term of PSNR values for test images is approximately $2.5 \mathrm{~dB}$.

Table 1. PSNR Values of Different Methods.

\begin{tabular}{cccccccc}
\hline $\begin{array}{c}\text { Noise } \\
\text { Level }\end{array}$ & $\begin{array}{c}\text { Noisy } \\
\text { Image }\end{array}$ & Wavelet & $\begin{array}{c}\text { Curvelet } \\
\text { hard }\end{array}$ & $\begin{array}{c}\text { BLS-GS } \\
\text { M }\end{array}$ & GSCE & $\begin{array}{c}\text { Curvelet } \\
\text { MAP }\end{array}$ & $\begin{array}{c}\text { Contourlet } \\
\text { MAP }\end{array}$ \\
\hline$\sigma_{n}=10$ & 28.19 & 28.47 & 29.28 & 33.08 & 31.71 & 32.18 & 34.63 \\
$\sigma_{n}=20$ & 22.13 & 25.49 & 26.09 & 28.93 & 28.09 & 28.29 & 31.40 \\
$\sigma_{n}=30$ & 18.64 & 24.56 & 24.33 & 26.78 & 26.07 & 26.18 & 28.31 \\
\hline
\end{tabular}




\section{Conclusions}

This paper presents an effective and useful beyond wavelets based image denoising method, which combines Bayesian MAP estimator rule. A lot of experimental results show that the proposed method surpasses the state-of-the-art methods.

\section{References}

(1) Y. Li, H. Lu, S. Serikawa: "Non-Gaussian Multivariate Shrinkage Function-basd image Denoising Method in Contourlet Domain", Proceeding of the Sixth International Conference on Information, vol.3, no.5, pp.997-1004, 2013

(2) R. Cohen: "Signal Denoising Using Wavelets", Project Report, 2012

(3) J.L. Starck, E.J. Candes, D.L. Donoho: "The Curvelet Transform for Image Denoising”, IEEE Transactions on Image Processing, vol.11, no.6, pp.670-684, 2002

(4) L. Zhang, Y. Li: "Anisotropic Multivariate Shrinkage Function-Based Image Denoising in the Second Generation Curvelet Transform Domain", ICIC Express Letters, Part B: Applications, vol.3, no.5, pp.1133-1138, 2012

(5) Q. Guo, S. Yu: "Image Denoising Using A Multivariate Shrinkage Function in the Curvelet Domain", IEICE Elec.Expr., vol.7, no.3, pp.126-131, 2010

(6) M.N. Do, M. Vetterli: "The Contourlet Transform: An Efficient Directional Multiresolution Image Representation", IEEE Trans. Image Process., vol.14, no.12, pp.2091-2106, 2005

(7) H. Lu, Y. Li, S. Serikawa: "Underwater Laser Image Denoising Using Bayesian Contourlet Estimator of Bessel K Form", ICIC Express Letters, Part B: Applications, vol.4, no.3, pp.533-539, 2013

(8) H. Lu, L. Zhang, S. Serikawa: "Maximum local energy: an effective approach for image fusion in beyond wavelet transform domain", Computers and Mathematics with Application, vol.64, no.5, pp.996-1003, 2012. 